\title{
Three-dimensional gravity-capillary solitary waves in water of finite depth and related problems
}

\author{
E.I. Părău, J.-M. Vanden-Broeck, ${ }^{*}$ and M.J. Cooker \\ School of Mathematics, University of East Anglia, Norwich, NR4 7TJ, UK
}

(Dated: 11 July 2005)

\begin{abstract}
Numerical solutions for three-dimensional gravity capillary waves in water of finite depth are presented. The full Euler equations are used and the waves are calculated by a boundary integral equation method. The findings generalise previous results of E. I. Parau, J.-M. Vanden-Broeck and M.J. Cooker [J. Fluid Mech. 536, 99 (2005)] in water of infinite depth. It is found that there are both lumps which bifurcate from linear sinusoidal waves and other fully localised solitary waves which exist for large values of the Bond number. These findings are consistent with rigorous analytical results and asymptotic calculations. The relation between the solitary waves and free surface flows generated by moving disturbances is also explored.
\end{abstract}

PACS numbers: 47.20.Ky; 47.35.+i 


\section{INTRODUCTION}

Two-dimensional gravity solitary waves have been investigated analytically and numerically since the pioneering work of Korteweg and de Vries. ${ }^{1}$ The study of two-dimensional gravity capillary solitary waves is more recent although Korteweg and de Vries already included surface tension in the derivation of their classical equation.

It was found both numerically and analytically that two-dimensional gravity solitary waves develop in the far field an oscillatory tail of constant amplitude as soon as a small amount of surface tension is introduced (see Hunter and Vanden-Broeck ${ }^{2}$, Champneys et al ${ }^{3}$ for numerical calculations and Dias and $\operatorname{Iooss}^{4}$ for a review of analytical work). These waves are often referred to as generalised solitary waves to contrast them from true solitary waves which are flat in the far field.

Other studies have shown that there are also two-dimensional gravity capillary solitary waves with decaying oscillatory tails in the far field. These waves exist both in water of finite depth and in water of infinite depth. ${ }^{5-7}$

In a previous paper $^{8}$, we computed three-dimensional gravity capillary solitary waves in water of infinite depth for the full Euler equations. These waves are left-right and fore-aft symmetric, have decaying oscillatory tails in the direction of propagation but have monotonic decay in the direction perpendicular to that of propagation. They can be viewed as the three-dimensional equivalent of the the two-dimensional gravity capillary solitary waves with decaying oscillatory tails described in the previous paragraph.

Here we consider three-dimensional gravity-capillary solitary waves in water of finite depth. These waves have been considered recently by Kim and Akylas ${ }^{9}$ and Milewski ${ }^{10}$ who derived weakly nonlinear models, and by Groves and Sun ("Fully localised solitarywave solutions of the three-dimensional gravity-capillary water-wave problem", preprint) who proved rigorously their existence. We show that there are two types of solutions. One type is similar to those obtained in water of infinite depth in the sense that the waves approach those in infinite depth in the limit as the depth tends to infinity. The other type has profiles with monotonic decay in all directions and exists only for sufficiently large values of the Bond number.

Introductory analytical results based on the dispersion relation are discussed in Section II. The boundary integral equation method is described in Section III and results are presented 
in Section IV. Finally we show in Section V that some free surface flows generated by moving disturbances can be viewed as perturbations of three-dimensional solitary waves.

\section{DISPERSION RELATION}

The classical problem of three-dimensional water waves in finite depth when both gravity and surface tension are present is considered. Some insight into the problem can be gained from the dispersion relation for linearized capillary-gravity waves travelling at a constant velocity $c$ in water of finite depth $h$. It can be written as (see for instance Lamb, ${ }^{11}$ Wehausen and Laitone ${ }^{12}$ )

$$
D(\kappa ; \lambda, \beta) \equiv\left(\lambda+\beta \kappa^{2}\right) \tanh \kappa-\kappa=0
$$

where $\kappa=k^{*} h$ is the dimensionless wavenumber. This equation involves two dimensionless numbers

$$
\lambda=\frac{g h}{c^{2}} \quad \text { and } \beta=\frac{T}{\rho h c^{2}},
$$

where $T$ is the constant coefficient of surface tension, $g$ is the acceleration due to gravity, $\rho$ is the fluid density. The parameter $\lambda$ is the inverse of the square of the Froude number and both parameters are related to the Bond number $B=T / \rho g h^{2}$ by the relation $B=\beta / \lambda$.

Kim and Akylas $^{9}$ have shown that three-dimensional solitary waves (which they call lumps) can bifurcate from linear sinusoidal waves with wavenumber corresponding to the minimum of the phase speed which is also a double root of the dispersion relation (1). This minimum corresponds, in the plane $(\beta, \lambda)$, to a a curve $\Gamma$, given in parametric form (see Dias and $\operatorname{Iooss}^{6}$ ) by

$$
\begin{aligned}
\beta & =\frac{1}{2 \kappa \sinh ^{2} \kappa}(\sinh \kappa \cosh \kappa-\kappa), \\
\lambda & =\frac{\kappa}{2 \sinh ^{2} \kappa}(\sinh \kappa \cosh \kappa+\kappa) .
\end{aligned}
$$

In the limit as $\kappa$ approaches zero, $\beta \sim 1 / 3-2 \kappa^{2} / 45, \lambda \sim 1+\kappa^{4} / 45$ and as $\kappa$ approaches infinity, $\beta \sim 1 /(2 \kappa), \lambda \sim \kappa / 2$. The curve is shown in Fig. 1. Lumps are predicted to exist on the region above the curve $\Gamma$. It is worth noting that two-dimensional gravity-capillary waves are shown to exist in the same region of parameters. ${ }^{13}$ Results similar to those of Kim and Akylas ${ }^{9}$ were obtained by Milewski ${ }^{10}$ for a weakly nonlinear model equation. 
Groves and Sun have shown that fully localised solitary waves also exist for another set of parameters

$$
\beta>1 / 3, \quad 0<\lambda-1 \ll 1
$$

In this region of strong surface tension Kadomtsev and Petviashvili ${ }^{14}$ derived the well-known KP-I equation as a long-wave approximation for solutions of the steady gravity-capillary water wave problem which has fully localized solitary-wave solutions.

The results of Kim and Akylas ${ }^{9}$ and Milewski ${ }^{10}$ are restricted to the weakly nonlinear regime. Here we present fully nonlinear computations of the waves described in the two previous paragraphs.

\section{FORMULATION AND NUMERICAL SCHEME}

The fluid is assumed to be incompressible and inviscid, and the flow to be irrotational. We are interested in steady waves which travel at a constant velocity $c$ in water of finite depth $h$, and we choose a frame of reference moving with the wave, so that the flow is steady. We introduce cartesian coordinates $x, y, z$ with the $z$-axis directed vertically upwards and the $x$-axis in the direction of wave propagation. We denote by $z=\zeta(x, y)$ the equation of the free surface. Dimensionless variables are introduced by taking the unit length to be $T / \rho c^{2}$ and the unit velocity to be $c$. In terms of the velocity potential function $\Phi(x, y, z)$, the problem is formulated as follows:

$$
\nabla^{2} \Phi=0, \quad x, y \in \mathbf{R},-\frac{1}{\beta}<z<\zeta(x, y)
$$

with the boundary conditions

$$
\begin{gathered}
\Phi_{x} \zeta_{x}+\Phi_{y} \zeta_{y}=\Phi_{z}, \quad \text { on } z=\zeta(x, y) \\
\frac{1}{2}\left(\Phi_{x}^{2}+\Phi_{y}^{2}+\Phi_{z}^{2}\right)+\lambda \beta \zeta- \\
-\left[\frac{\zeta_{x}}{\sqrt{1+\zeta_{x}^{2}+\zeta_{y}^{2}}}\right]_{x}-\left[\frac{\zeta_{y}}{\sqrt{1+\zeta_{x}^{2}+\zeta_{y}^{2}}}\right]_{y}=\frac{1}{2}, \quad \text { on } z=\zeta(x, y),
\end{gathered}
$$

and

$$
\Phi_{z}=0, \quad \text { on } z=-\frac{1}{\beta} .
$$


Equations (5) and (7) are the kinematic boundary conditions on the free surface and on the bottom. Equation (6) is the dynamic condition with the effect of surface tension included.

Since we are looking for fully localised three-dimensional solitary waves, we impose the conditions

$$
\left(\Phi_{x}, \Phi_{y}, \Phi_{z}\right) \rightarrow(1,0,0), \zeta \rightarrow 0, \text { as } \quad\left(x^{2}+y^{2}\right)^{1 / 2} \rightarrow \infty
$$

to fix the value of Bernoulli's constant in (6).

The numerical scheme is an extension to finite depth of the scheme used by Părău, Vanden-Broeck and Cooker ${ }^{8}$ for the computation of the fully localised gravity-capillary waves in deep water and it is based on a boundary integral equation method introduced by Forbes ${ }^{15}$ for three dimensional gravity free surface flows due to a source. Only the main points of the formulation and of the numerical procedure are presented here. The reader is referred to Părău and Vanden-Broeck ${ }^{16}$ for details.

The formulation involves applying Green's second identity for the functions $\Phi-x$ and $G$ where $G$ is the three dimensional free space Green function

$$
G=\frac{1}{4 \pi} \frac{1}{\left(\left(x-x^{*}\right)^{2}+\left(y-y^{*}\right)^{2}+\left(z-z^{*}\right)^{2}\right)^{1 / 2}},
$$

for a volume $V$ which consists of a cylinder bounded by the free surface (except a small hemisphere around the point $P\left(x^{*}, y^{*}, z^{*}\right)$ ), and its image $S_{F^{\prime}}$ on the other side of the bottom $z=-1 / \beta$ (see Fig. 2). In that way, by symmetry, the condition of no flow normal to the bottom (7) is satisfied. This technique was applied for the problem of a withdrawal through a point sink by Hocking, Vanden-Broeck and Forbes ${ }^{17}$ and others.

After some manipulation of the surface integrals and after projecting them onto the $O x y$ plane, we obtain

$$
\begin{gathered}
\frac{1}{2}\left(\phi\left(x^{*}, y^{*}\right)-x^{*}\right)= \\
=\iint_{\mathbf{R}^{2}}(\phi(x, y)-x) \frac{1}{4 \pi} \frac{\zeta(x, y)-\zeta\left(x^{*}, y^{*}\right)-\left(x-x^{*}\right) \zeta_{x}(x, y)-\left(y-y^{*}\right) \zeta_{y}(x, y)}{\left(\left(x-x^{*}\right)^{2}+\left(y-y^{*}\right)^{2}+\left(\zeta(x, y)-\zeta\left(x^{*}, y^{*}\right)\right)^{2}\right)^{3 / 2}} d x d y+ \\
+\iint_{\mathbf{R}^{2}} \frac{1}{4 \pi} \frac{\zeta_{x}(x, y)}{\left(\left(x-x^{*}\right)^{2}+\left(y-y^{*}\right)^{2}+\left(\zeta(x, y)-\zeta\left(x^{*}, y^{*}\right)\right)^{2}\right)^{1 / 2}} d x d y+ \\
+\iint_{\mathbf{R}^{2}}(\phi(x, y)-x) \frac{1}{4 \pi} \frac{\zeta(x, y)+\zeta\left(x^{*}, y^{*}\right)+2 / \beta-\left(x-x^{*}\right) \zeta_{x}(x, y)-\left(y-y^{*}\right) \zeta_{y}(x, y)}{\left(\left(x-x^{*}\right)^{2}+\left(y-y^{*}\right)^{2}+\left(\zeta(x, y)+\zeta\left(x^{*}, y^{*}\right)+2 / \beta\right)^{2}\right)^{3 / 2}} d x d y+ \\
+\iint_{\mathbf{R}^{2}} \frac{1}{4 \pi} \frac{\zeta_{x}(x, y)}{\left(\left(x-x^{*}\right)^{2}+\left(y-y^{*}\right)^{2}+\left(\zeta(x, y)+\zeta\left(x^{*}, y^{*}\right)+2 / \beta\right)^{2}\right)^{1 / 2}} d x d y
\end{gathered}
$$


where $\phi(x, y)=\Phi(x, y, \zeta(x, y))$.

We look for solutions which are symmetric in $x$ and $y$. Therefore we assume

$$
\begin{gathered}
\zeta(x, y)=\zeta(-x, y)=\zeta(x,-y)=\zeta(-x,-y), \\
\phi(x, y)=-\phi(-x, y)=\phi(x,-y)=-\phi(-x,-y) .
\end{gathered}
$$

Following Forbes ${ }^{15}$, Părău and Vanden-Broeck ${ }^{16}$ and Părău et al. ${ }^{8}$ we rewrite equation (10) as

$$
2 \pi\left(\phi\left(x^{*}, y^{*}\right)-x^{*}\right)=I_{1}+I_{2}+I_{3}+I_{4}
$$

where

$$
\begin{aligned}
& I_{1}=\int_{0}^{\infty} \int_{0}^{\infty}\left[\left(\phi(x, y)-\phi\left(x^{*}, y^{*}\right)-x+x^{*}\right) K_{1 a}\left(x, y, x^{*}, y^{*}\right)+\right. \\
& \left.+\left(-\phi(x, y)-\phi\left(x^{*}, y^{*}\right)+x+x^{*}\right) K_{1 b}\left(x, y, x^{*}, y^{*}\right)\right] d x d y \\
& I_{2}=\int_{0}^{\infty} \int_{0}^{\infty}\left(\zeta_{x}(x, y) K_{2 a}\left(x, y, x^{*}, y^{*}\right)-\zeta_{x}(x, y) K_{2 b}\left(x, y, x^{*}, y^{*}\right)\right) d x d y \\
& I_{3}=\int_{0}^{\infty} \int_{0}^{\infty}\left[(\phi(x, y)-x) K_{3 a}\left(x, y, x^{*}, y^{*}\right)+\right. \\
& \left.+(-\phi(x, y)+x) K_{3 b}\left(x, y, x^{*}, y^{*}\right)\right] d x d y \\
& I_{4}=\int_{0}^{\infty} \int_{0}^{\infty}\left(\zeta_{x}(x, y) K_{4 a}\left(x, y, x^{*}, y^{*}\right)-\zeta_{x}(x, y) K_{4 b}\left(x, y, x^{*}, y^{*}\right)\right) d x d y, \\
& K_{1 a}\left(x, y, x^{*}, y^{*}\right)=\frac{\zeta(x, y)-\zeta\left(x^{*}, y^{*}\right)-\left(x-x^{*}\right) \zeta_{x}(x, y)-\left(y-y^{*}\right) \zeta_{y}(x, y)}{\left(\left(x-x^{*}\right)^{2}+\left(y-y^{*}\right)^{2}+\left(\zeta(x, y)-\zeta\left(x^{*}, y^{*}\right)\right)^{2}\right)^{3 / 2}}+ \\
& +\frac{\zeta(x, y)-\zeta\left(x^{*}, y^{*}\right)-\left(x-x^{*}\right) \zeta_{x}(x, y)-\left(y+y^{*}\right) \zeta_{y}(x, y)}{\left(\left(x-x^{*}\right)^{2}+\left(y+y^{*}\right)^{2}+\left(\zeta(x, y)-\zeta\left(x^{*}, y^{*}\right)\right)^{2}\right)^{3 / 2}}, \\
& K_{2 a}\left(x, y, x^{*}, y^{*}\right)=\frac{1}{\sqrt{\left(x-x^{*}\right)^{2}+\left(y-y^{*}\right)^{2}+\left(\zeta(x, y)-\zeta\left(x^{*}, y^{*}\right)\right)^{2}}}+ \\
& +\frac{1}{\sqrt{\left(x-x^{*}\right)^{2}+\left(y+y^{*}\right)^{2}+\left(\zeta(x, y)-\zeta\left(x^{*}, y^{*}\right)\right)^{2}}}, \\
& K_{3 a}\left(x, y, x^{*}, y^{*}\right)=\frac{\zeta(x, y)+\zeta\left(x^{*}, y^{*}\right)+2 / \beta-\left(x-x^{*}\right) \zeta_{x}(x, y)-\left(y-y^{*}\right) \zeta_{y}(x, y)}{\left(\left(x-x^{*}\right)^{2}+\left(y-y^{*}\right)^{2}+\left(\zeta(x, y)+\zeta\left(x^{*}, y^{*}\right)+2 / \beta\right)^{2}\right)^{3 / 2}}+ \\
& +\frac{\zeta(x, y)+\zeta\left(x^{*}, y^{*}\right)+2 / \beta-\left(x-x^{*}\right) \zeta_{x}(x, y)-\left(y+y^{*}\right) \zeta_{y}(x, y)}{\left(\left(x-x^{*}\right)^{2}+\left(y+y^{*}\right)^{2}+\left(\zeta(x, y)+\zeta\left(x^{*}, y^{*}\right)+2 / \beta\right)^{2}\right)^{3 / 2}}, \\
& K_{4 a}\left(x, y, x^{*}, y^{*}\right)=\frac{1}{\sqrt{\left(x-x^{*}\right)^{2}+\left(y-y^{*}\right)^{2}+\left(\zeta(x, y)+\zeta\left(x^{*}, y^{*}\right)+2 / \beta\right)^{2}}}+
\end{aligned}
$$




$$
+\frac{1}{\sqrt{\left(x-x^{*}\right)^{2}+\left(y+y^{*}\right)^{2}+\left(\zeta(x, y)+\zeta\left(x^{*}, y^{*}\right)+2 / \beta\right)^{2}}}
$$

and $K_{i b}\left(x, y, x^{*}, y^{*}\right)=K_{i a}\left(-x, y, x^{*}, y^{*}\right), i=1, . ., 4$.

We note that the integrand in $I_{2}$ is singular whereas those in $I_{1}, I_{3}$ and $I_{4}$ are not.

We truncate the intervals $0<x<\infty$ and $0<y<\infty$ to $x_{1}<x<x_{N}$, and $y_{1}<y<y_{M}$ and introduce the mesh points $x_{i}=(i-1) \Delta x, i=1, \ldots, N$ and $y_{j}=(j-1) \Delta y, j=1, \ldots, M$. The integral $I_{2}$ (which contains the singularity) can be calculated using some indefinite integral (see Forbes ${ }^{15}$ ). The $2 N M$ unknowns are

$$
\mathbf{u}=\left(\zeta_{x_{11}}, \zeta_{x_{12}}, \ldots, \zeta_{x_{N, M-1}}, \zeta_{x_{N M}}, \phi_{11}, \ldots, \phi_{N M}\right)^{T}
$$

where we use the notation $\zeta_{x_{i j}}=\zeta_{x}\left(x_{i}, y_{j}\right), \phi_{i j}=\phi\left(x_{i}, y_{j}\right), \zeta_{i j}=\zeta\left(x_{i}, y_{j}\right)$ etc.

The integrals and the Bernoulli equation are evaluated at the points $\left(x_{i+1 / 2}, y_{j}\right), i=$ $1, \ldots, N-2, j=1, \ldots, M$ so we have $2(N-2) M$ equations. Another $2 M$ equations are obtained from the truncation conditions $\zeta_{x_{N j}}=0, \phi_{x_{N j}}=1, j=1, \ldots, M$ and another $2 M$ equations are given by the symmetry conditions $\zeta_{x_{1 j}}=0$ and by $\phi_{1 j}=0$. The values of $\zeta$ are obtained by integrating $\zeta_{x}$ with respect to $x$ by the trapezoidal rule. The values of $\phi_{x}$, $\zeta_{y}$ and $\phi_{y}$ are then calculated by central differences. The values of the variables $\zeta$ and $\phi$ at $\left(x_{i+1 / 2}, y_{j}\right)$ were obtained by interpolation and the values of the other derivatives which appears in the problem were computed by finite differences.

The $2 N M$ nonlinear equations are solved by Newton's method. Most of the computations were performed with $\Delta x=\Delta y=0.8$ and $N=40, M=50$. The accuracy of the solutions have been tested by varying the number of grid points and the intervals $\Delta x$ and $\Delta y$ between grid points.

The formulation in infinite depth was given in Părău, Vanden-Broeck and Cooker ${ }^{8}$ and was obtained by applying Green's second identity on a volume bounded by the free surface and a half sphere of arbitrarily large radius in the fluid. The main difference from the present case is that equation (11) does not contain the integrals $I_{3}$ and $I_{4}$. We also use the parameter

$$
\alpha=\frac{g T}{\rho c^{4}}
$$

instead of the term $\lambda \beta$ in the equation (6), since we cannot use the parameters $\lambda$ and $\beta$, which are based on $h$. 
Generally, in order to obtain a solitary-wave solution in finite depth we first compute forced gravity-capillary waves for fixed $\lambda$ and $\beta$ (see Section $\mathrm{V}$ ), then we remove gradually the pressure, by keeping the amplitude of the solution constant. The solution obtaned is then used as an initial guess or further computations.

\section{GRAVITY-CAPILLARY SOLITARY WAVES}

For small surface tension $(\beta<1 / 3)$ we found that the three dimensional problem is qualitatively similar to the two dimensional problem. In particular there are two branches of fully localised three-dimensional gravity-capillary solitary waves. One branch has a central depression, the other branch has a central elevation. These waves have decaying oscillations in the direction of propagation and are monotonically decaying perpendicular to the direction of propagation. In Fig.3 we show a typical central depression gravity-capillary wave solution and in Fig.4 a typical central elevation gravity-capillary wave solution. The curves obtained by cutting the free surface with planes parallel to the direction of propagation are qualitatively similar to the two dimensional profiles obtained by Vanden-Broeck and Dias ${ }^{5}$ and by Dias et al. ${ }^{7}$. The solutions are quite similar to the fully localised solitary waves found on deep water (see Parau et al. ${ }^{8}$ ).

For strong surface tension $(\beta>1 / 3)$ we found only fully localised depression gravity capillary solitary waves. They are similar to fully-localized solitary-wave solutions of the KP-I equation as shown in Fig. 5 for $\lambda=1.14$ and $\beta=1$. The lump solution for the KP-I equation is, as given by Milewski ${ }^{10}$ and rewritten in term of our parameters, is

$$
\zeta(x, y)=\frac{-16 \mu}{\beta} \frac{3-2 \frac{\mu}{\nu} \beta^{2} x^{2}+4 \frac{\mu^{2}}{\nu} \beta^{2} y^{2}}{\left(3+2 \frac{\mu}{\nu} \beta^{2} x^{2}+4 \frac{\mu^{2}}{\nu} \beta^{2} y^{2}\right)^{2}}
$$

where $\mu=1-\frac{1}{\sqrt{\lambda}}$ and $\nu=\frac{\beta}{\lambda}-\frac{1}{3}$. Figure 6 shows the $x$ and $y$ cross-section of the computed fully-localized solitary-wave solution (solid line) and the lump solution for the KP-I equation (dotted line). It can be observed that there is a very good agreement between the two solutions for the centreline on the $O x$ direction, except at the last points, where the effect of the truncation is visible. There is also a good agreement on the $O y$ direction between the numerical and analytical solution, but the numerical solution seems to decay faster than the analytical solution. This is likely to be caused by truncation, as the algebraic decay of the solution in this direction is slower than on the $O x$ direction. 
The agreement between the amplitude of the KP-I solution and our solution of the full equations can be observed better if we keep $\lambda$ constant and vary $\beta$ (see Fig. 7). We can follow continuously a branch of central depression solitary-wave solutions from the region of strong surface tension to the region of weak surface tension, by passing through $\beta=1 / 3$, as predicted by Milewski ${ }^{10}$. There is a maximum amplitude for the branch of central depression waves (with $\lambda$ constant) for $\beta$ near $1 / 3$.

\section{FORCED CAPILLARY-GRAVITY WAVES}

In this section we consider three-dimensional free surface flows due to a moving disturbance. As we shall see some of the solutions can be viewed as perturbations of the solitary waves of Section III. We choose a frame of reference moving with the disturbance and we seek steady solutions. The disturbance can be submerged (e.g. a submarine) or surface piercing (e.g. a ship or a probe). The results presented are qualitatively independent of the disturbance chosen. Therefore we assume for simplicity that the disturbance is a distribution of pressure $\varepsilon P(x, y)$ where $\varepsilon$ is a given parameter and

$$
P(x, y)=\left\{\begin{array}{cc}
e^{\frac{1}{\left(x^{2}-1\right)}+\frac{1}{\left(y^{2}-1\right)},} & |x|<1 \text { and }|y|<1 \\
0, & \text { otherwise }
\end{array}\right.
$$

The problem is described mathematically by the equations (4)-(7) with the term $\epsilon P(x, y)$ added on the left hand side of (6). For finite depth numerical solutions are calculated by using the numerical procedure of Section III. For infinite depth the numerical scheme is similar to that in Părău, Vanden-Broeck and Cooker ${ }^{8}$.

We calculated solutions both in finite and infinite depth and obtained qualitatively similar results. In Fig. 8 and 9 we present two such profiles for $\lambda=1.132$ and $\beta=1$. The first one is obtained by imposing a positive pressure $(\varepsilon>0)$ on the surface and the second one by imposing a negative pressure distribution $(\varepsilon<0)$. To simplify the problem, we will present from now on results obtained in infinite depth only, as the profiles are quite similar to the ones in finite depth and the number of parameters is decreased by one ( $\alpha$ and $\varepsilon$ ).

In all cases $\alpha$ is assumed to be greater than $1 / 4$, which corresponds to flows where the distribution of pressure moves steadily with a constant velocity $c$ smaller than the minimum 
phase speed $c_{\text {min }}$, which in infinite depth is

$$
c_{\text {min }}=\left(\frac{4 g T}{\rho}\right)^{1 / 4} .
$$

In this case no wave pattern was observed and only a highly localised disturbance of the water surface is predicted. As $\alpha$ decreases and approaches $1 / 4$ more and more oscillations appear in front and behind the main disturbance, the area disturbed by the pressure increasing considerably. In two dimensions, Vanden-Broeck \& Dias ${ }^{5}$ computed capillary-gravity waves in the same regime of parameters $(\alpha>1 / 4)$.

We show in Figure 10 values of $\zeta(0,0)$ versus $\alpha$ for various values of $\varepsilon$. The dashed lines corresponds to $\varepsilon= \pm 1$. For each $\varepsilon \neq 0$, there is a critical number $\alpha_{\varepsilon}$ such that there is no solution for $1 / 4<\alpha<\alpha_{\varepsilon}$. In order to compute solutions near the turning point we used a variation of the scheme of Section III in which the amplitude is fixed and $\alpha$ is found as part of the solution.

Linear solutions for the flow due to a moving pressure distribution were calculated by Raphaël and de Gennes ${ }^{18}$, Sun and Keller ${ }^{19}$ and others. We have recalculated these solutions numerically by running the code with the boundary conditions linearized. The corresponding values of $\zeta(0,0)$ versus $\alpha$ are shown in Figure 10 by dotted lines. It shows that $|\zeta(0,0)| \rightarrow \infty$ as $\alpha \rightarrow 1 / 4$. Therefore the linear theory is not valid near $\alpha=1 / 4$. Our nonlinear solutions do not blow up as $\alpha \rightarrow 1 / 4$ but have a turning point at $\alpha=\alpha_{e}>1 / 4$. The solutions corresponding to the portions of the broken curves closest to the $\alpha$-axis and extending from $\alpha_{e}$ to $\alpha=\infty$ are close to the linear solutions corresponding to the dotted lines in Figure 10. These solutions are perturbations of a uniform stream in the sense that they approach a uniform stream with constant velocity $U$ as $|\epsilon| \rightarrow 0$.

The remaining portions of the broken curves (i.e. the portions of the curves further away from the $\alpha$-axis and extending to the right of the turning point $\alpha_{e}$ ) are perturbations of three-dimensional gravity-capillary solitary waves. In other words the remaining portions of the broken curves approach solitary waves as $|\epsilon| \rightarrow 0$. The remaining portion of the broken curve for $\epsilon=1$ is already almost on the branch of depression solitary waves. The remaining portion for $\epsilon=-1$ is still not very close to the branch of elevation solitary waves, but it approaches it when $|\epsilon|$ decreases.

The branches of solitary waves correspond to the solid curves in Figure 10 and they were discussed in detail in Parau et al. ${ }^{8}$. 
Figure 10 shows forced solutions that are elevation solitary waves perturbed by a negative distribution of pressure $(\epsilon<0)$ and forced solutions that are depression solitary waves perturbed by a positive distribution of pressure $(\epsilon>0)$. We found that there are also forced capillary gravity waves that are elevation solitary waves perturbed by positive pressure distributions and depression solitary waves perturbed by negative pressure distributions. A typical example is shown in Figure 11.

\section{CONCLUSION}

We have calculated three dimensional gravity capillary solitary waves of the full Euler equations in finite depth. Two branches of solutions were obtained. One is bifurcating at the minimum value of the phase speed of the linear periodic waves. There are both elevation and depression waves. The other one is an extension of the solution of the KP-I equation to the fully nonlinear regime. We have shown that some of the solutions corresponding to flows due to moving disturbances can be viewed as perturbations of these solitary waves.

\section{Acknowledgments}

This work was supported by EPSRC, under Grant Number GR/S47786/01.

* Author to whom the correspondence should be addressed. Electronic mail: J.Vandenbroeck@uea.ac.uk

1 D.J. Korteweg and G. de Vries, "On the Change of Form of Long Waves advancing in a Rectangular Canal and on a New Type of Long Stationary Waves," Philosophical Magazine 36, 422 (1895).

2 J.K. Hunter and J.-M. Vanden-Broeck, "Solitary and periodic gravity-capillary waves of finite amplitude," J. Fluid Mech. 134, 205 (1983).

3 A.R.Champneys, J.-M. Vanden-Broeck and G.J. Lord, "Do true elevation gravity-capillary solitary waves exist? A numerical investigation," J. Fluid Mech. 454, 403 (2002).

4 F.Dias, G.Iooss, "Water-waves as a spatial dynamical system." In Handbook of Mathematical Fluid Dynamics, Vol. 2, 443 (Elsevier, Amsterdam, 2003). . 
5 J.-M. Vanden-Broeck and F. Dias "Gravity-capillary solitary waves in water of infinite depth and related free-surface flows," J. Fluid Mech., 240, 549 (1992).

6 F. Dias and G. Iooss, "Capillary-gravity solitary waves with damped oscillations", Physica D 65, 399 (1993).

7 F. Dias, D. Menasce and J.-M. Vanden-Broeck "Numerical study of capillary-gravity solitary waves," Eur. J. Mech., B/Fluids, 15, 17 (1996).

8 E. Părău, J.-M. Vanden-Broeck, M. Cooker, "Nonlinear three dimensional gravity-capillary solitary waves and related free-surface flows," J. Fluid Mech. 536, 99 (2005).

9 B. Kim and T.R. Akylas, "On gravity-capillary lumps," J. Fluid Mech. 540, 337 (2005).

10 P.A. Milewski, "Thee-dimensional localized solitary gravity-capillary waves," Commun. Math. Sci. 3, 89 (2005).

11 H. Lamb Hydrodynamics, 6th Edn. (Cambridge University Press, Cambridge, 1932).

12 J.W. Wehausen and E.V. Laitone, "Surface Waves". In Encyclopaedia of Physics Vol. IX, 446 (Springer Verlag, Berlin, 1960).

13 G. Iooss and K. Kirchgässner, "Water waves for small surface tension: an approach via normal form”, Proc. R. Soc. Edinburgh A 122, 267 (1992).

14 B.B. Kadomtsev and V.I. Petviashvili, "On the stability of solitary waves in weakly dispersing media," Sov. Phys. Dokll. 15, 539 (1970).

15 L.K. Forbes, "An algorithm for 3-dimensional free-surface problems in hydrodynamics," J. Comp. Physics 82, 330 (1989).

16 E. Părău and J.-M. Vanden-Broeck, "Nonlinear two- and three-dimensional free surface flows due to moving disturbances," Eur. J. Mechanics B/Fluids, 21, 643 (2002).

17 G.C. Hocking, J.-M. Vanden-Broeck and L.K. Forbes, "A note on withdrawal from a fluid of finite depth through a point sink", ANZIAM J. 44, 181 (2002).

18 E. Raphäel, P.-G. de Gennes "Capillary gravity waves caused by a moving disturbance. Wave resistance," Physical Review E, 53, 3448 (1996).

19 S.-M. Sun, J.B. Keller, "Capillary-gravity wave drag," Phys. Fluids, 13, 2146 (2001). 
FIG. 1. Curves in the $\beta-\lambda$ plane where solitary waves can bifurcate (the grey area): above the curve $\Gamma$ for $\beta<1 / 3$ and above the curve $\lambda=1$ for $\beta<1 / 3$. The curve $\Gamma$ is shown as a dark solid line.

FIG. 2. The surfaces used for the application of the Green's second identity.

FIG. 3. Central depression solitary gravity-capillary wave for $\beta=0.235, \lambda=1.13$. Only half of the solutions $(y \geq 0)$ are shown.

FIG. 4. Central elevation solitary gravity-capillary wave for $\beta=0.28, \lambda=1.13$. Only half of the solutions $(y \geq 0)$ are shown.

FIG. 5. Central depression solitary gravity-capillary wave for $\beta=1, \lambda=1.14(x \leq 0)$ compared with the fully localised solitary wave solution for KP-I $(x \geq 0)$, as given by Milewski ${ }^{10}$. Only half of the solutions $(y \geq 0)$ are shown.

FIG. 6. (a) The centreline in the $O x$ direction of the computed solitary gravity-capillary wave (solid line) and the fully localised solitary wave solution for KP-I (dotted line). The parameters are $\beta=1, \lambda=1.14$. (b) The centreline in the $O y$ direction of the computed solitary gravity-capillary wave (solid line) and the fully localised solitary wave solution for KP-I (dotted line). The parameters are $\beta=1, \lambda=1.14$.

FIG. 7. The maximum amplitude of the computed solution (solid line) and KP-I solutions (dashed line) for a fixed $\lambda=0.132$

FIG. 8. Forced capillary-gravity waves for $\lambda=1.132, \beta=1$ and $\varepsilon=0.3$.

FIG. 9. Forced capillary-gravity waves for $\lambda=1.132, \beta=1$ and $\varepsilon=-0.3$.

FIG. 10. Values of the amplitude $(\zeta(0,0))$ versus $\alpha$. The dashed lines corresponds to $\varepsilon=1$ in the negative half of the plane and to $\varepsilon=-1$ on the positive half of the plane. The solid line corresponds to free capillary-gravity waves $(\varepsilon=0)$. The dotted line corresponds to linear solutions for $\varepsilon= \pm 1$.

FIG. 11. Free surface profile for $\alpha=0.35, \varepsilon=3$ which is a perturbation of an elevation solitary wave. Only half of the solution is shown. 


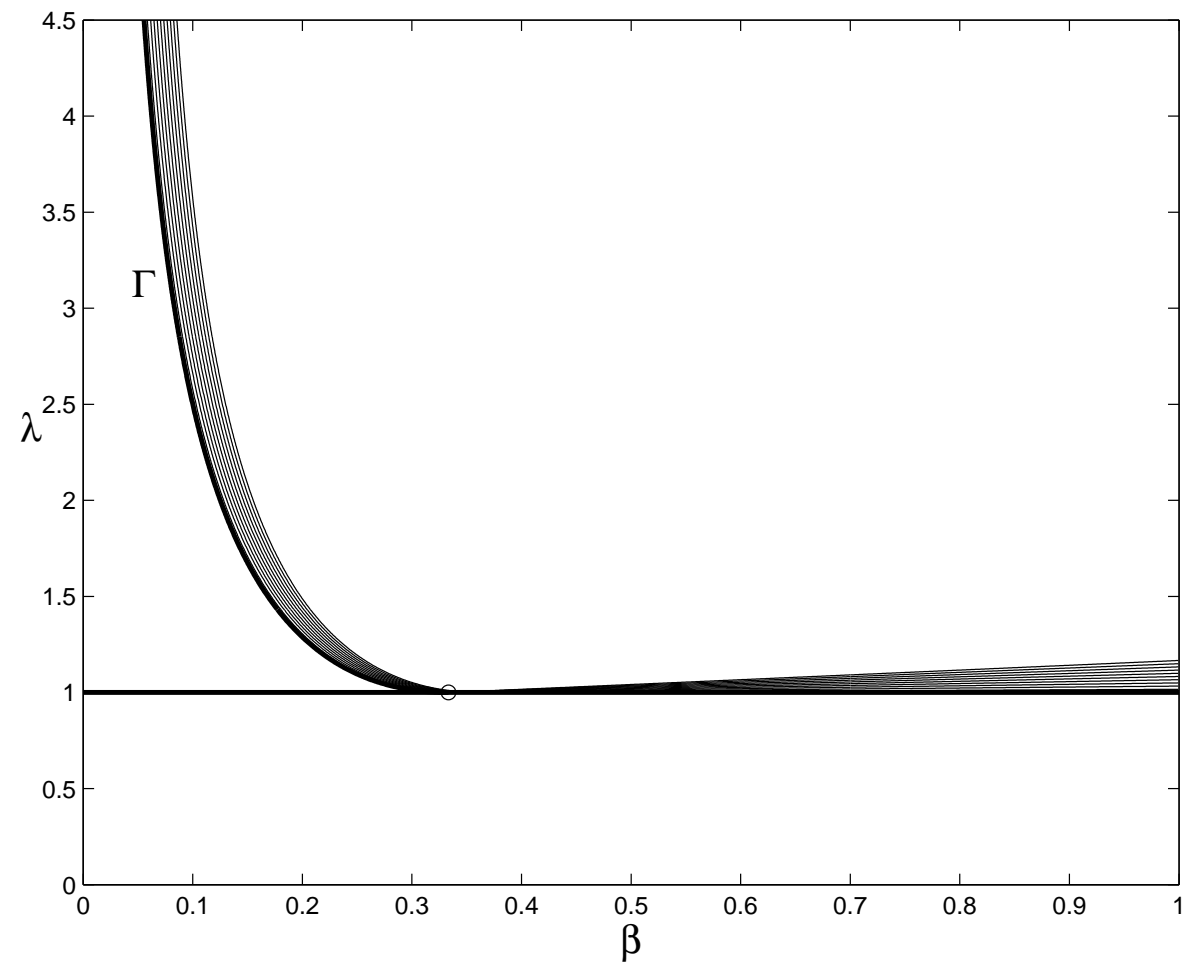

FIG. 1: Parau, Physics of Fluids 


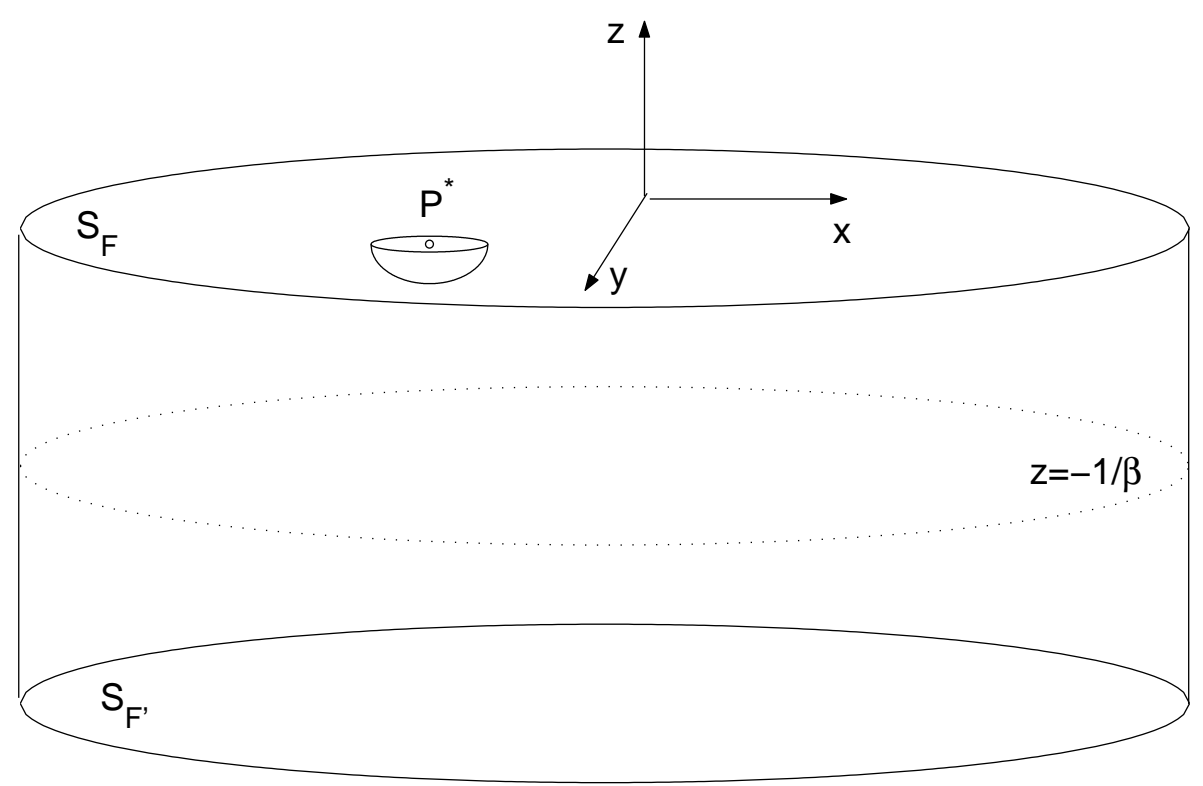

FIG. 2: Parau, Physics of Fluids 


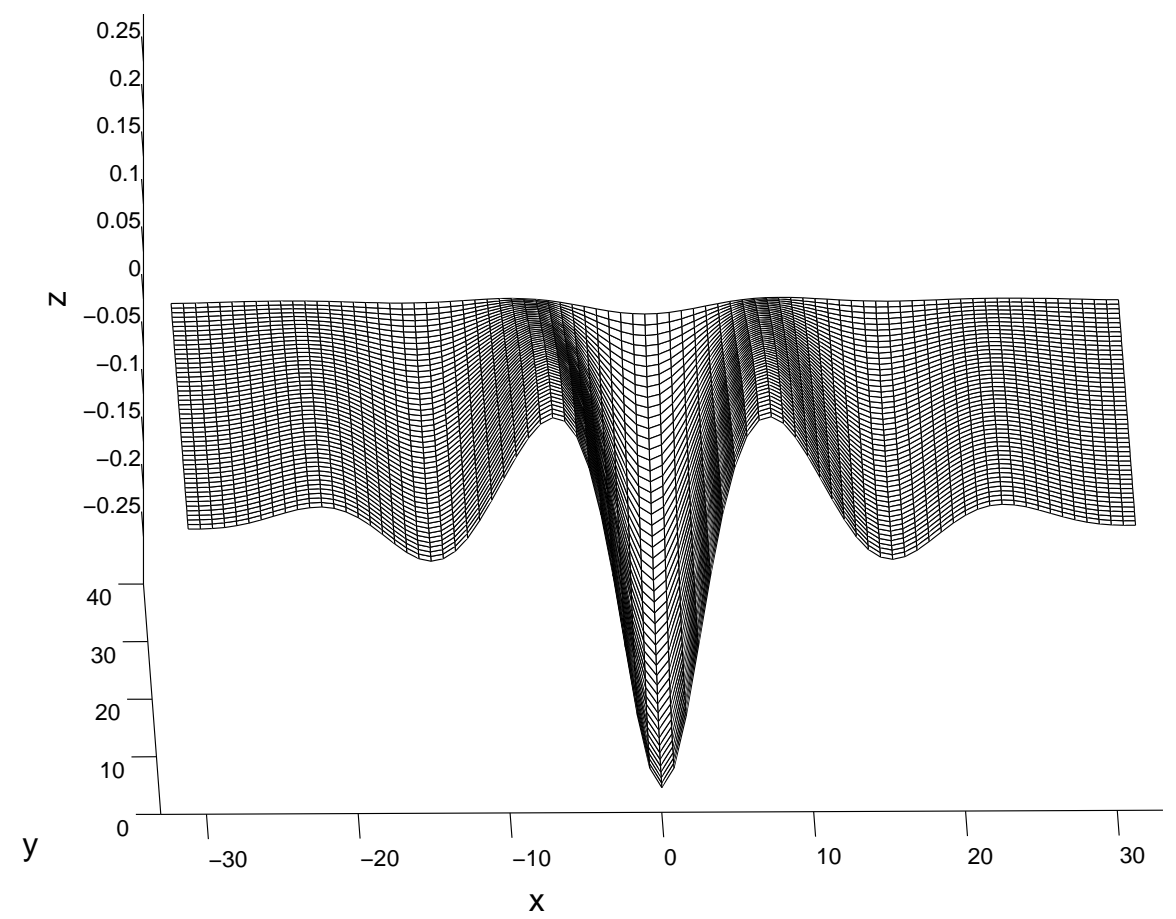

FIG. 3: Parau, Physics of Fluids 


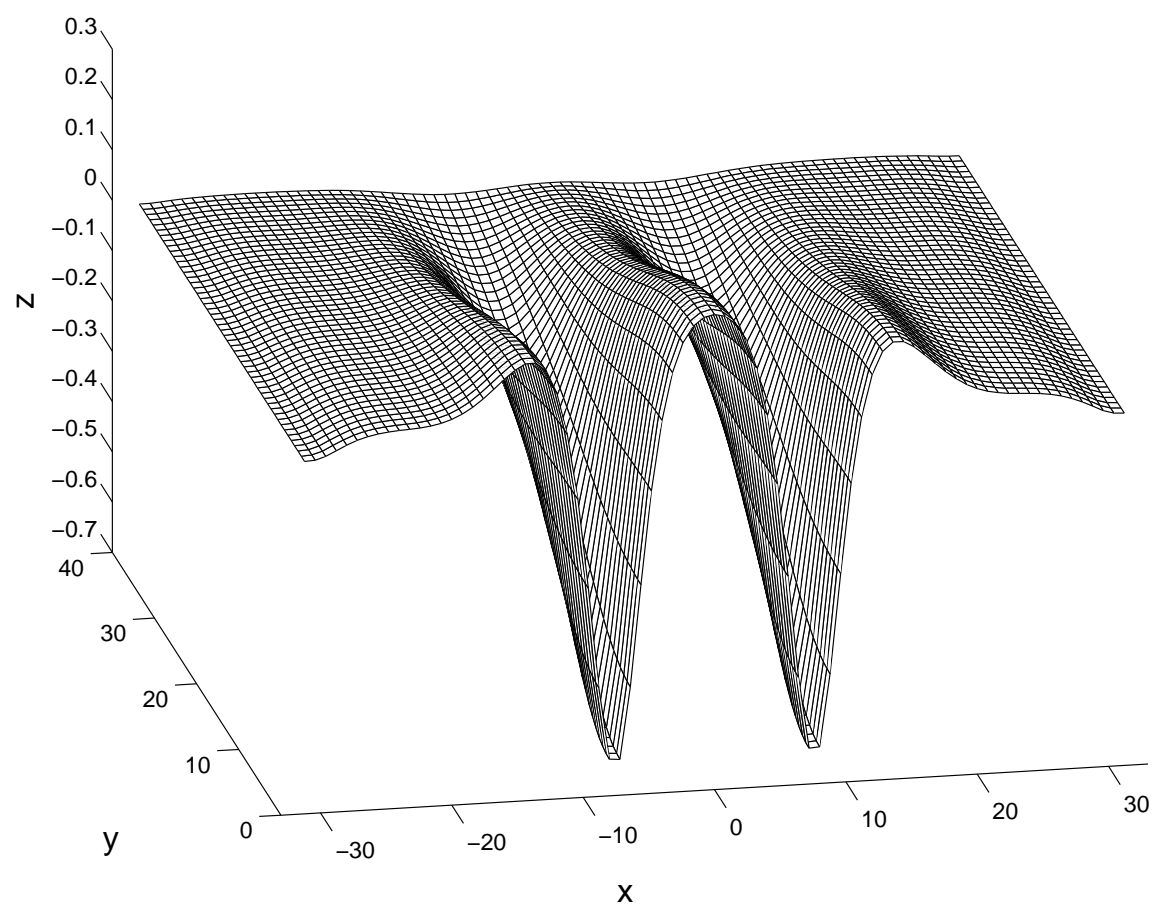

FIG. 4: Parau, Physics of Fluids 


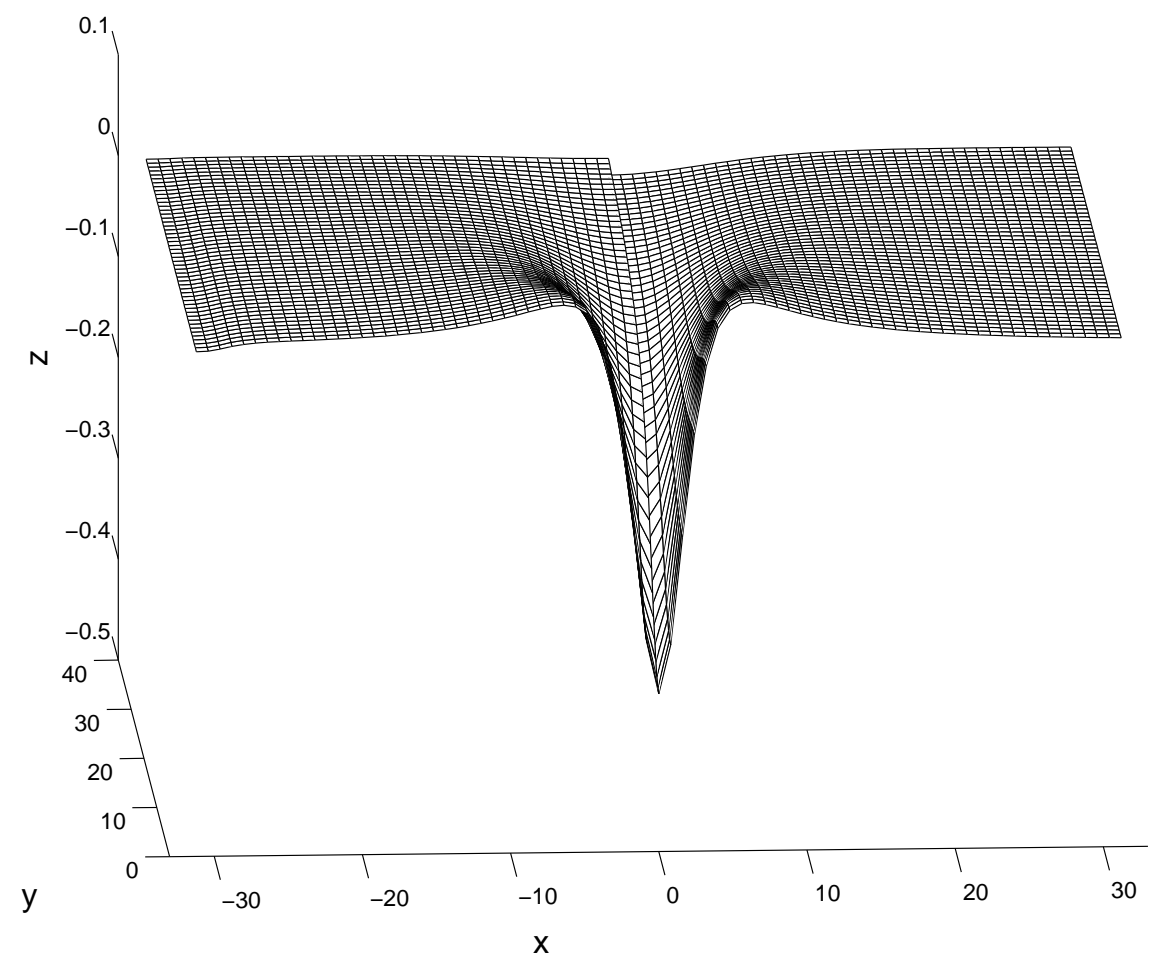

FIG. 5: Parau, Vanden-Broeck, Cooker 
(a)

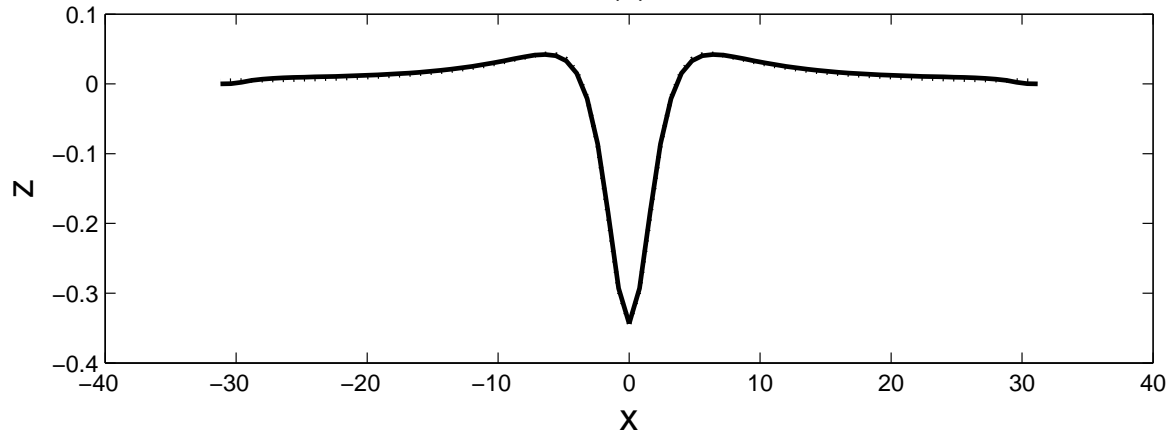

(b)

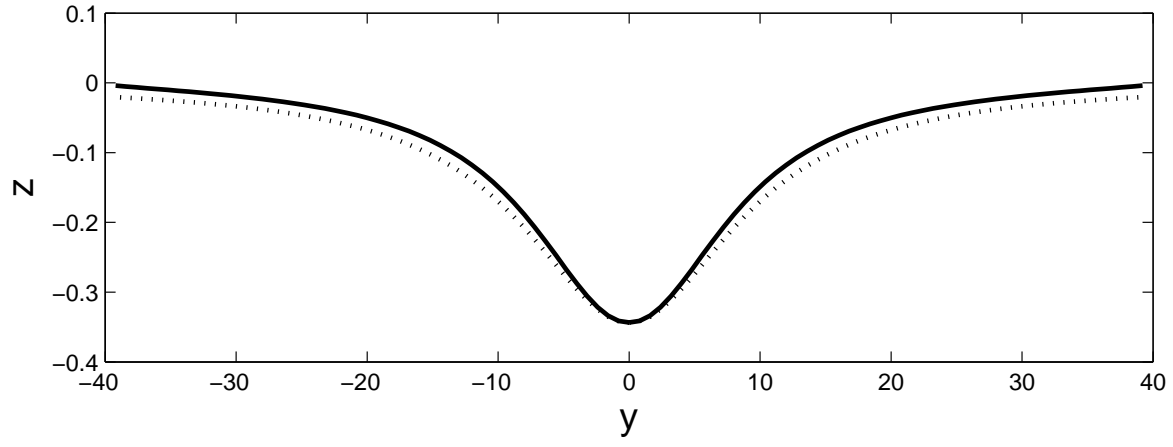

FIG. 6: Parau, Physics of Fluids 


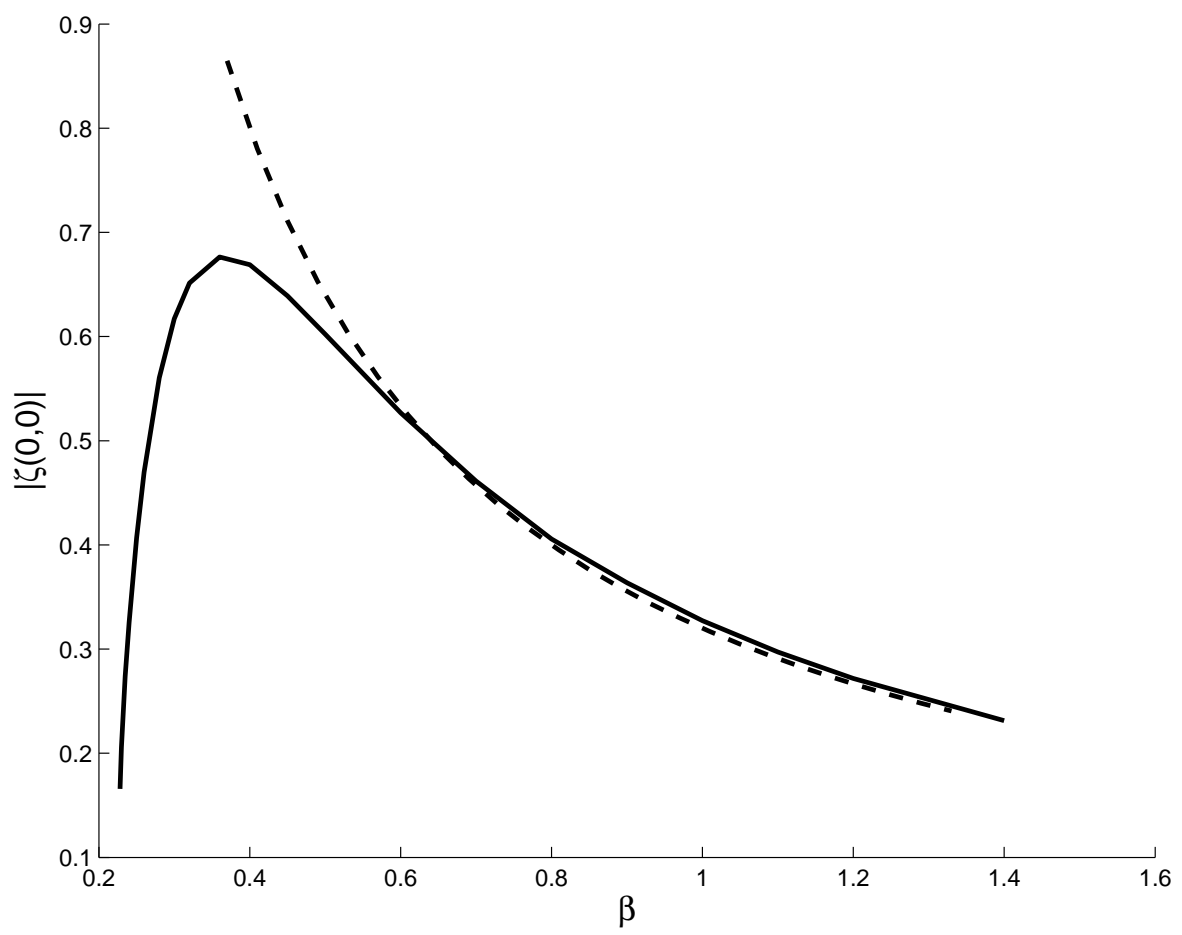

FIG. 7: Parau, Physics of Fluids 


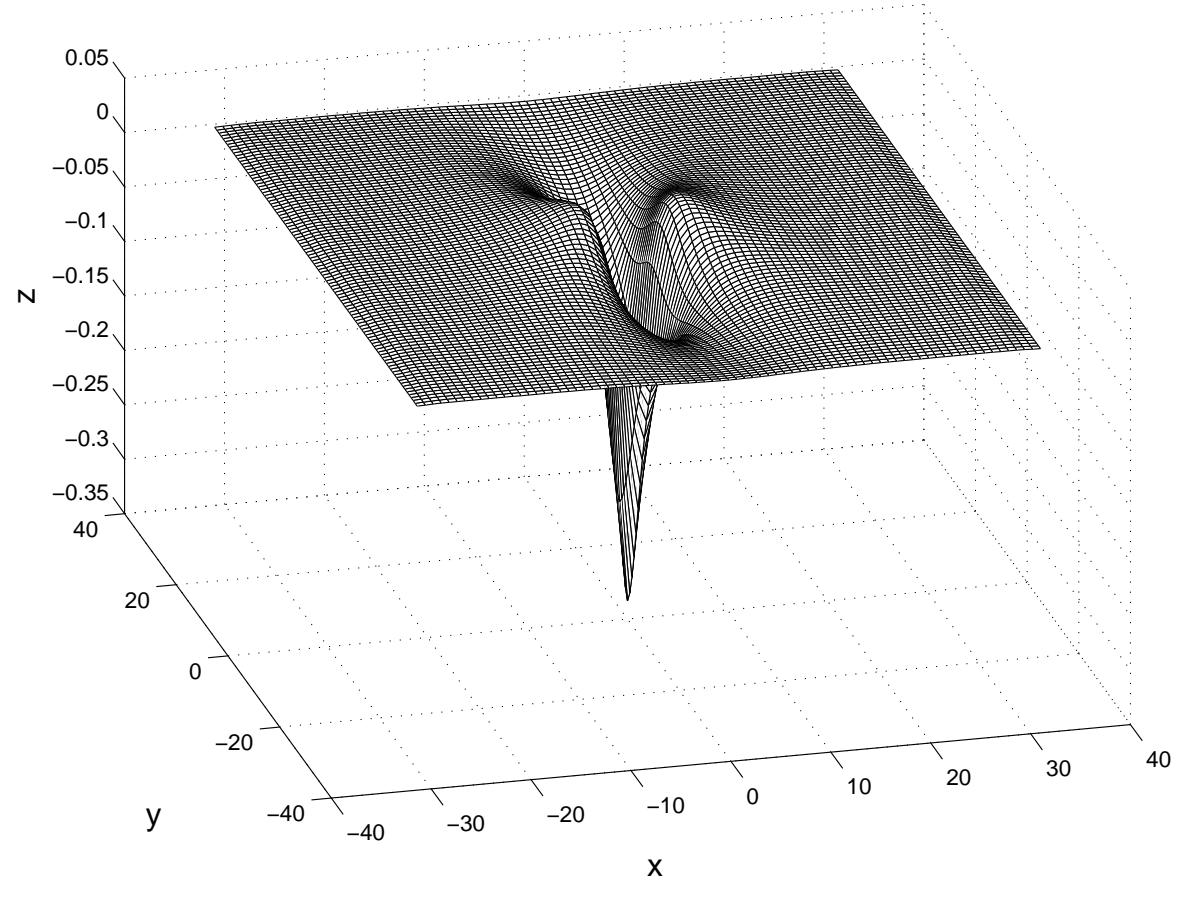

FIG. 8: Parau, Physics of Fluids 


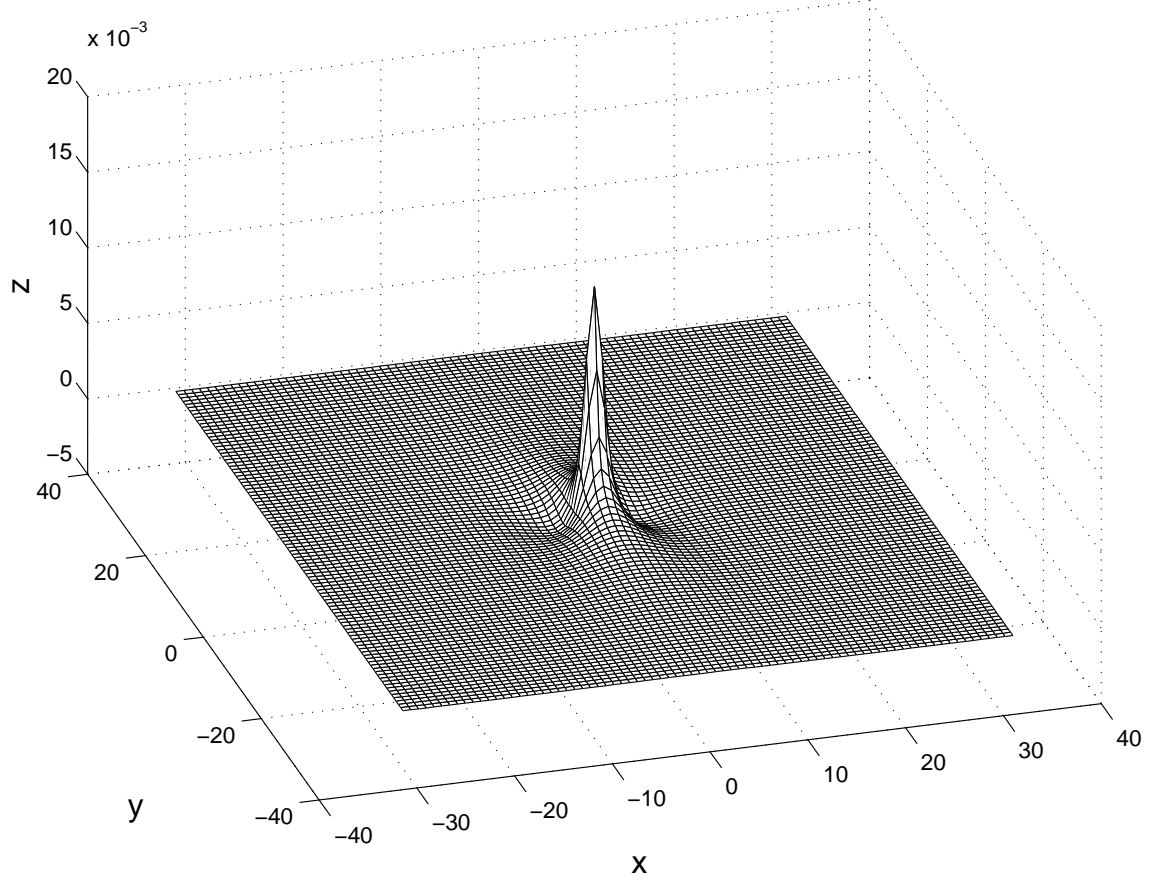

FIG. 9: Parau, Physics of Fluids

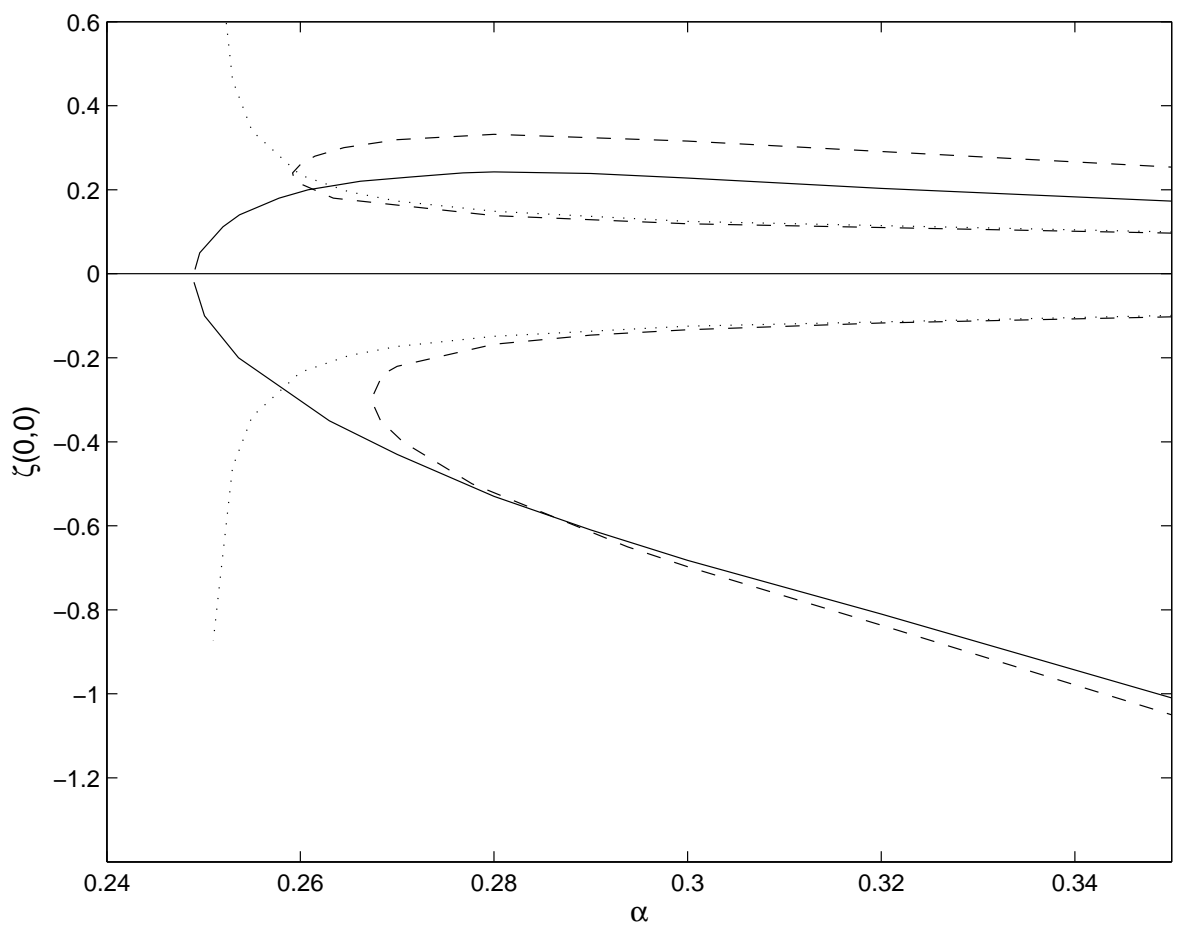

FIG. 10: Parau, Physics of Fluids 


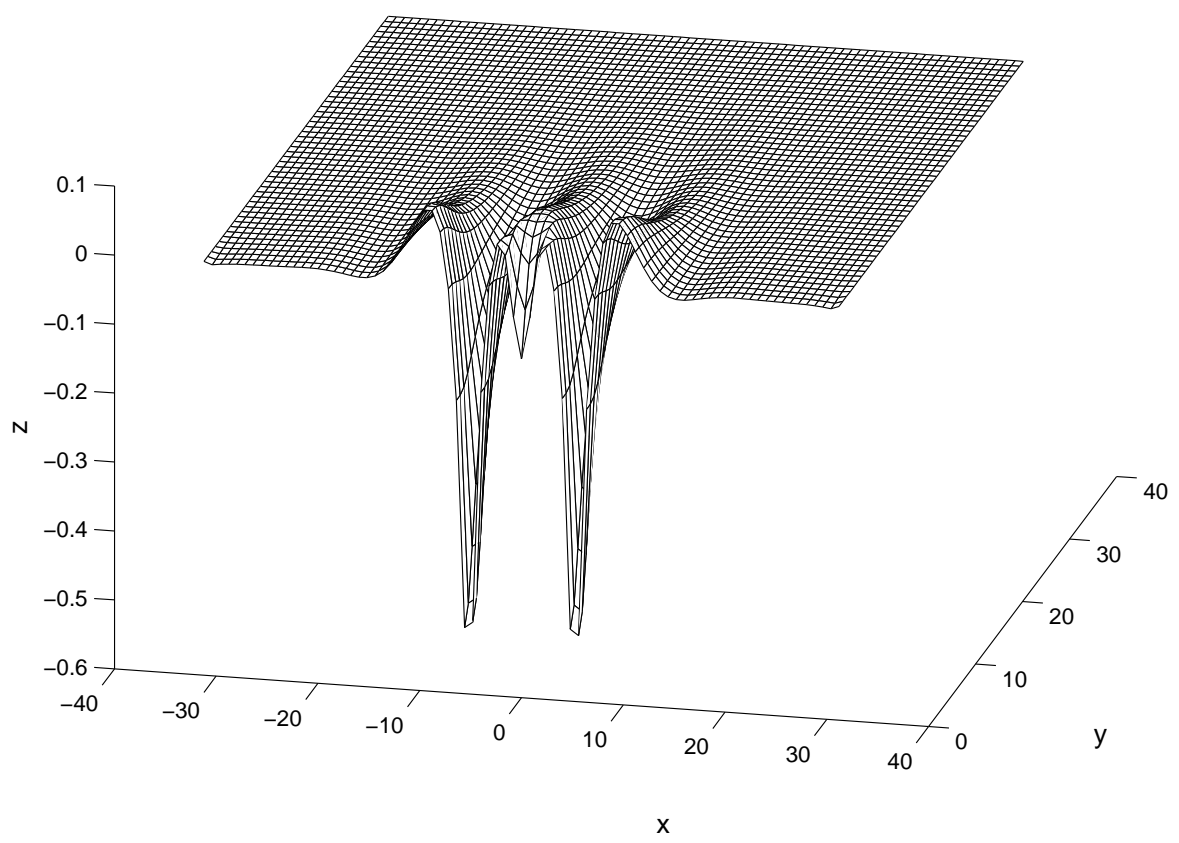

FIG. 11: Parau, Physics of Fluids 